\title{
State-of-charge indication in Li-ion batteries by simulated impedance spectroscopy
}

\author{
Shruti Srivastav $^{1} \cdot$ Matthew J. Lacey $^{1} \cdot$ Daniel Brandell $^{1}$
}

Received: 24 September 2016/ Accepted: 8 December 2016/Published online: 22 December 2016

(c) The Author(s) 2016. This article is published with open access at Springerlink.com

\begin{abstract}
We here explore the possibilities of correlating experimental cell impedance with finite element methodology modelling for state-of-charge $(\mathrm{SoC})$ indication in $\mathrm{LiFePO}_{4}$-based half-cells. The impedance response has been modelled sequentially during battery cycling using Newman theory, and is compared with experimental data. It is found that the charge-transfer resistance is dependent of SoC during battery charging, which can be modelled in good agreement with experimental results. Moreover, it is seen that cell design parameters-e.g. calendering-dependent electrode porosity-influence the EIS response and can thus be estimated using the presented methodology.
\end{abstract}

Daniel Brandell

daniel.brandell@kemi.uu.se

1 Department of Chemistry- Ångström Laboratory, Uppsala University, Box 538, 75121 Uppsala, Sweden

\section{Graphical Abstract}

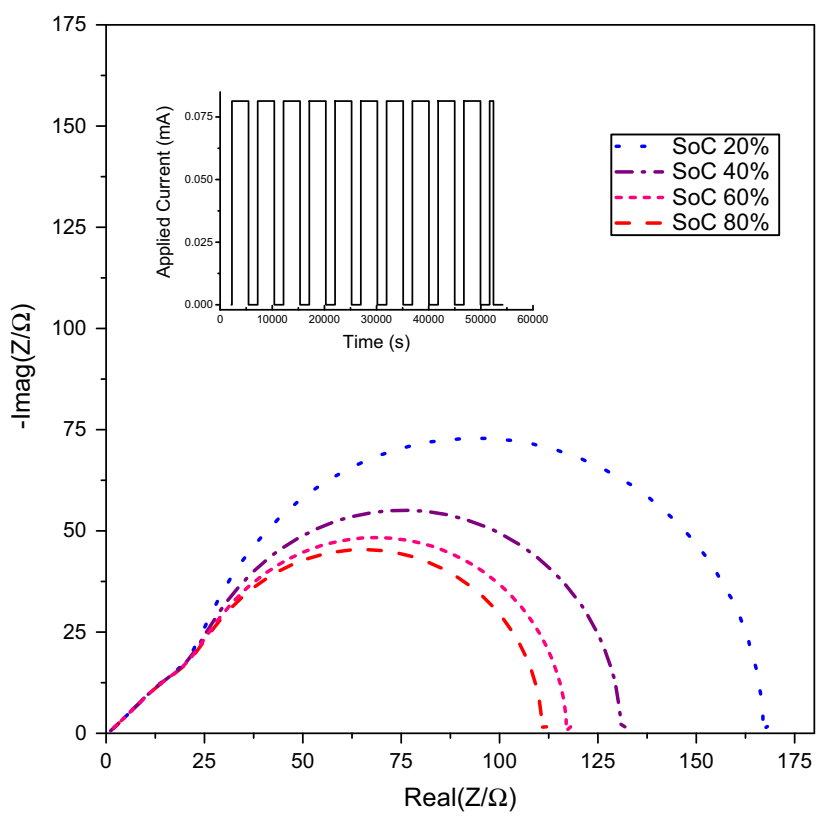

Keywords Li-ion battery $\cdot$ State-of-charge determination . Impedance spectroscopy $\cdot$ Finite element methodology

\section{Introduction}

As the utilization of large-scale batteries is currently increasing, so does the demand for more accurate battery diagnostics, thereby generating a rapid development of different battery management systems (BMSs) $[1,2]$. Here, the state-of-charge ( $\mathrm{SoC})$ is one of the most critical parameters for indication of the remaining energy left in a 
cell. Developing efficient yet accurate SoC algorithms remains a challenge. Electrochemical impedance spectroscopy (EIS) is in this context considered a fast, nondestructive and reliable method. EIS can be used to determine important parameters such as charge-transfer resistance and double-layer capacitance, from which information on battery utilization can be derived [2, 3]. By extracting electrical parameters which vary as a function of the battery SoC, the impedance spectra can be used as a predictive tool. Generally, only the high-frequency response is used to estimate internal resistance and thereby SoC [3].

The measured total impedance constitutes the result of a large number of resistive components and reactive processes, which are usually assessed by an equivalent-circuit analogue. This circuit represents, for example, equilibrium potential, ohmic behaviour, charge transfer, double-layer effects at the electrode/electrolyte interfaces and mass transfer processes. In present battery diagnostics, both simple lumped-parameter circuits and more complex finitetransmission-line circuits are used [4-6]. However, most of the proposed electric equivalent models do not represent the electrochemical phenomena at each of the electrodes, but globally at cell level, thus generating an oversimplification. Moreover, porous-electrode effects, transient and non-linear responses, and additional artefacts of the battery current collectors, terminals, and other more peripheral components are not assessable by equivalent-circuit modelling. The contributions of these physically distinct processes are instead merged into one EIS response, rendering a meaningful interpretation less possible.

Since the impedance spectra of batteries strongly depend on the short-term charge/discharge history, the validity of equivalent-circuit modelling is restricted. To overcome this problem, we suggest an approach that combines EIS with a physics-based cell model. Finite element methodology (FEM) has been a valuable approach for modelling batteries with a range of cell types and geometries [7-10]. A FEM approach allows for a model based on physical properties of the system-such as thermodynamics, kinetics and diffusion-as opposed to simplified circuit elements representing these processes. In principle, such a model can be extended to three-dimensional models of complete cells or battery systems.

However, this approach has so far not been widely applied to exploring factors affecting SoC in Li-ion batteries (LiBs). In one recent example, such an approach is used for commercial LiB cells with reasonable accuracy [11], but is limited due to the restricted information on the electrode formulation and morphology. In this work, we attempt to explore the possibilities of correlating the impedance of lab-scale half-cells with controlled chemistry to their SoC, using FEM modelling and direct comparisons of the simulated EIS results with experimental analogues, also including electrode morphological effects.

The principal aim of this study is to investigate if this combination of FEM with impedance spectroscopy is a feasible approach for battery SOC diagnostics, and also to pinpoint some of the current limitations with such an approach.

\section{Finite element studies}

FEM studies have proven fruitful for battery modelling. Many of the mathematical models use porous-electrode theory [12], thereby treating the electrodes as a uniform mix of active material, binder, conducting additive and electrolyte. Wang and Sastry [13], however, implemented concentrated solution theory using FEM to break down the electrode material mix in order to study the influence of size distribution of the active material particles. Similarly, FEM has proven useful for simulating the impedance response of complex energy storage devices [14]. However, the physical processes and properties influencing the impedance response and its correlation to the $\mathrm{SoC}$ in a $\mathrm{LiB}$ are still to a large degree unexplored. In this work, we attempt to investigate the possibilities of correlating cell impedance and SoC using FEM modelling. To achieve this, we have used the COMSOL Multiphysics batteries and fuel cells module (BFC) to construct a $1 \mathrm{D}$ model of a $\mathrm{LiB}$, which incorporates morphological properties such as porosity in the determination of the impedance spectra. Experimental data are used to validate the simulations.

\subsection{Model background}

A lithium half-cell with a lithium-iron phosphate (LiFe$\mathrm{PO}_{4}$ ) positive electrode has been modelled using the BFC module in COMSOL Multiphysics 4.4, simulating the battery charging profile. 1D half-cell lithium battery model consists of the cell cross section in one dimension, implying that edge effects in length and height are neglected. The cell is constituted in three regions (two electrodes and a separator) rendering four distinct boundaries at

(a) $0<x<\delta_{\text {cc }}$ : Positive current collector (aluminium)

(b) $\delta_{\mathrm{cc}}<x<\delta_{\mathrm{p}}$ : Positive porous electrode $\left(\mathrm{LiFePO}_{4}\right)$. Thickness: $25 \mu \mathrm{m}$

(c) $\delta_{\mathrm{p}}<x<\delta_{\mathrm{s}}$ : Separator $(1 \mathrm{M} \mathrm{LiPF} 6$ salt in $1: 1$ EC:DEC as electrolyte). Thickness: $50 \mu \mathrm{m}$

(d) $\delta_{\mathrm{s}}<x<\delta_{\mathrm{n}}$ : Counter-electrode (lithium foil). Thickness: $25 \mu \mathrm{m}$.

For the electronic current balance, a potential of $0 \mathrm{~V}$ is set on the counter electrode boundary $\left(x=\delta_{\mathrm{n}}\right)$. At the working 
electrode current collector/feeder $\left(x=\delta_{\mathrm{cc}}\right)$, the current density (harmonic perturbation) is specified. The inner boundaries facing the separator $x=\delta_{\mathrm{p}}$ and $x=\delta_{\mathrm{s}}$ ) are insulating for electric currents. To maintain the ionic charge balance in the electrolyte, the current collector boundaries $\left(x=\delta_{\mathrm{cc}}\right.$ and $\left.x=\delta_{\mathrm{n}}\right)$ are ionically insulating. Insulating conditions also apply to the material balances.

\subsection{Electrochemical description}

The positive electrode is assumed porous. The region $0<x<\delta_{\mathrm{p}}$ therefore contains both solid porous-electrode and liquid (electrolyte) phases. At the particle surface, the material flux is determined by the local electrochemical reaction rate given by linearized Butler-Volmer kinetics. The electrical potential in the electron conducting phase $\left(\phi_{\mathrm{s}}\right)$ is calculated using a charge balance based on Ohm's law, where the charge-transfer reactions result in a mathematical source or sink term. Taking porosity and tortuosity into account, the effective conductivities of the electrodes $\left(\phi_{\text {eff }}\right)$ is given by

$\phi_{\mathrm{eff}}=\phi_{\mathrm{s}} \epsilon^{\gamma}$,

where $\gamma$ is the Bruggeman coefficient, set to 1.5 in this model, corresponding to a packed bed of porous particles. The ionic charge balances and material balances are modelled for binary 1:1 electrolytes. Fickian diffusion describes the mass transport of lithium ions to the particles (Eq. 2), whereby the resulting diffusion equation can be expressed from the concentration gradients of lithium ions in the porous electrode (Eq. 3). Butler-Volmer electrode kinetics describes the local charge-transfer current density in the electrodes and is introduced as source or sink terms in the charge balances and material balances (Eqs. 5-8).

Lithium diffusion in the spherical particle of radius " $r$ " is described by Fick's law according to [15]

$\frac{\partial}{\partial t} \delta C_{\mathrm{Li}}=\frac{D}{r^{2}} \frac{\partial}{\partial r}\left(r^{2} \frac{\partial C_{\mathrm{Li}}}{\partial r}\right)$

$\frac{\partial\left(\epsilon_{\mathrm{e}} C_{\mathrm{Li}}\right)}{\partial t}=\frac{\partial}{\partial x}\left(D_{\mathrm{Li}} \frac{\partial C_{\mathrm{Li}}}{\partial x}\right)+\frac{1-t_{\mathrm{Li}}^{0} j}{F} j$

with boundary conditions as

$\left.\frac{\partial C_{\mathrm{Li}}}{\partial x}\right|_{x=0}=\left.\frac{\partial C_{\mathrm{Li}}}{\partial x}\right|_{x=L}=0$.

Charge conservation in the solid phase of electrode is defined by Ohm's law:

$\frac{\partial}{\partial x}\left(\sigma_{\text {eff }} \frac{\partial}{\partial x} \phi_{\mathrm{s}}\right)-j=0$

with the following limit conditions at the current collectors $\left.\sigma_{+}^{\mathrm{eff}} \frac{\partial \phi_{\mathrm{s}}}{\partial x}\right|_{x=0}=\frac{I}{A}$

and the null current conditions at the separator:

$\left.\frac{\partial \phi_{\mathrm{s}}}{\partial x}\right|_{x=\delta_{\mathrm{n}}}=\left.\frac{\partial \phi_{\mathrm{s}}}{\partial x}\right|_{x=\delta_{\mathrm{n}}+\delta_{\mathrm{s}}}=0$.

If $\phi_{i}(x, t)$ denotes the electrolyte potential, charge conservation in the electrolyte is defined by

$\frac{\partial}{\partial x}\left(\kappa^{\mathrm{eff}} \frac{\partial}{\partial x} \phi_{i}\right)+\frac{\partial}{\partial x}\left(\kappa^{\mathrm{eff}} \frac{\partial}{\partial x} \ln \left(C_{i}\right)\right)+j=0$

with

$\left.\frac{\partial \phi_{i}}{\partial x}\right|_{x=0}=\left.\frac{\partial \phi_{i}}{\partial x}\right|_{x=L}=0$,

where $\kappa^{\text {eff }}$ is the conductivity in the electrolyte. The four differential Eqs. 2, 5, 6 and 8 are linked by the ButlerVolmer equation

$j / A=i_{0}\left(e^{\frac{a n F \eta}{R T}}-e^{\frac{(1-\alpha) \eta \eta \eta}{R T}}\right)$.

where $A$ is the electrode area. In Eq. 10, the total current $j$ is induced by overvoltage $\eta$, defined by the potential difference between the solid phase and the electrolyte and equilibrium thermodynamic potential $U$ :

$\eta=\phi_{\mathrm{s}}+\phi_{i}-U$,

where $U$ is a function of the degree of lithiation in the active material particles, and thus dependent on the SoC.

The admittance response (the inverse of the impedance) of the device is then generated through the diffusive flux of the charges species, and is related to the current through the following equations:

$$
\begin{aligned}
& Y(\omega)=\frac{l \omega}{\eta_{0}} \int_{0}^{\infty} \mathrm{d} t e^{-l \omega t} j(t) \\
& S o C(t)=\frac{1}{Q} \int_{0}^{t} j(t) \mathrm{d} t \\
& j(\omega) \propto Y(\omega)=\frac{l \omega}{\eta_{0}} \int_{0}^{\infty} \mathrm{d} t e^{-l \omega t} j(t) .
\end{aligned}
$$

These relations can, in turn, be related to Eqs. 10 and 12 , where the current passing through the battery is an indicator of the SoC. The relationship between impedance and $\mathrm{SoC}$ can thus be explored using this set of equations, and is here investigated by calculating the changing impedance spectra while incrementing the SoC with known intervals. Parameter values are listed in Table 1. 
Table 1 List of symbols and parameters

\begin{tabular}{lll}
\hline Variable & Value $(\mathrm{SI}$ units $)$ & Description \\
\hline$C_{\mathrm{Li}}$ & $\left(\mathrm{mol} / \mathrm{m}^{3}\right)$ & Li-ion concentration in active material \\
$D_{\mathrm{Li}}$ & $5 \times 10^{-13}\left(\mathrm{~m}^{2} / \mathrm{s}\right)$ & Li-ion diffusivity in active material \\
$r$ & $25 \times 10^{-8}(\mathrm{~m})$ & Particle radius of active material (positive electrode) \\
$T$ & $298(\mathrm{~K})$ & Temperature \\
$t_{\mathrm{l}}$ & 0.363 & Cation transport number \\
$D_{\mathrm{l}}$ & $7.5 \times 10^{-12}\left(\mathrm{~m}^{2} / \mathrm{s}\right)$ & Salt diffusivity in electrolyte \\
$\epsilon_{\mathrm{l}}$ & & Electrolyte volume fraction \\
$\epsilon_{\mathrm{S}}$ & $1-\epsilon_{\mathrm{l}}$ & Solid-phase volume fraction \\
$c_{l, 0}$ & $2000\left(\mathrm{~mol} / \mathrm{m}^{3}\right)$ & Initial electrolyte salt concentration \\
$k_{\mathrm{f}}$ & $2 \times 10^{-11}(\mathrm{~m} / \mathrm{s})$ & Forward reaction rate coefficient \\
$k_{\mathrm{b}}$ & $2 \times 10^{-11}(\mathrm{~m} / \mathrm{s})$ & Backward reaction rate coefficient \\
$\delta_{\mathrm{n}}$ & $25 \times 10^{-6}(\mathrm{~m})$ & Thickness of negative electrode \\
$\delta_{\mathrm{s}}$ & $50 \times 10^{-6}(\mathrm{~m})$ & Thickness of separator \\
$\delta_{\mathrm{p}}$ & $25 \times 10^{-6}(\mathrm{~m})$ & Thickness of positive electrode \\
$I$ & $(\mathrm{~A})$ & Applied current \\
$i_{\text {pert }}$ & $15\left(\mathrm{~mA} / \mathrm{cm}{ }^{2}\right)$ & Perturbation current \\
$i_{0}$ & $\left(\mathrm{~mA} / \mathrm{m}^{2}\right)$ & Exchange current density \\
$Y(\omega)$ & $(1 / \Omega)$ & Admittance \\
$\phi_{\mathrm{s}}$ & $(\mathrm{V})$ & Potential in the solid state phase \\
$\phi_{\mathrm{l}}$ & $(\mathrm{V})$ & Electrolyte potential \\
$\sigma_{\mathrm{eff}}$ & $(\mathrm{S} / \mathrm{m})$ & Effective electrical conductivity \\
$\kappa_{\mathrm{eff}}$ & $(\mathrm{S} / \mathrm{m})$ & Ionic conductivity of the electrolyte \\
$\omega$ & $(\mathrm{Hz})$ & Frequency \\
\hline & &
\end{tabular}

\subsection{Mesh density and solver settings}

Equations 1-14 have been solved using the COMSOL Multiphysics 5.1 software. A mesh length of $3.35 \times 10^{-4} \mathrm{~m}$ was used, and the device was divided into 47 mesh elements in total, using 4 vertex elements. The element length ratio has an average mesh growth rate of 1.083 . The same mesh was used in all simulations. A PARDISO solver with 0.01 relative and 0.001 absolute tolerances was used for the time-dependent matrix equations. A rectangular periodic pulse (inset in Fig. 1) with the same amplitude and period as employed in the experiments was used as the load for the applied current. By applying a prescribed load cycle, a time-dependent charging cycle was modelled. The flux was linearized at intervals of $30 \mathrm{mAh} / \mathrm{g}$ to yield the frequency domain response at different $\mathrm{SoC}$ levels using a harmonic perturbation of $15 \mathrm{mV}$.

\section{Experimental}

$\mathrm{LiFePO}_{4}$ (LFP, Phostech) was dispersed with carbon black (Super P, Imerys) and poly(vinylidene fluoride-co-hexafluoropropylene) (Kynar FLEX 2801, Arkema) in a ratio of $80: 15: 5$ by weight into $N$-methylpyrrolidone (NMP,

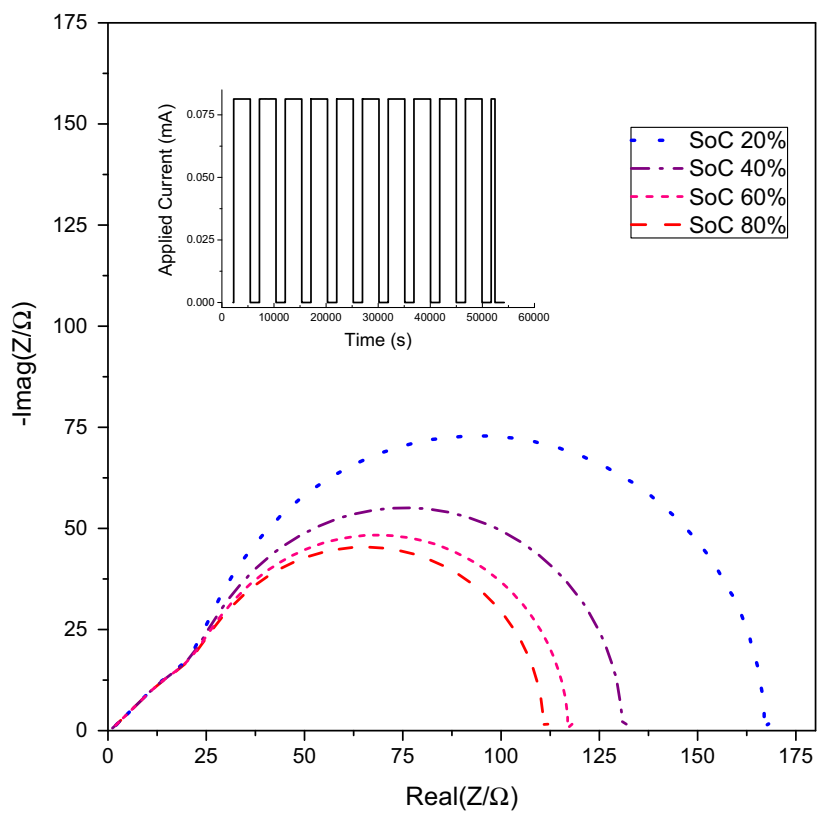

Fig. 1 Simulated Nyquist plots for various state-of-charge levels. Inset showing load-cycling

VWR) and mixed by planetary ball-milling for $1 \mathrm{~h}$. The resulting slurry was bar-coated onto $\mathrm{Al}$ foil and dried at 70 ${ }^{\circ} \mathrm{C}$ in air. The sheet was cut into 20 -mm-diameter discs, 
transferred to an Ar-filled glove box and dried at $120{ }^{\circ} \mathrm{C}$ under vacuum overnight. The LFP loading on the electrodes was $1.5 \mathrm{mg} / \mathrm{cm}^{2}$. Vacuum-sealed pouch-type halfcells were assembled with a 22-mm-diameter Li metal disc counter-electrode (Cyprus Foote Mineral, $125 \mu \mathrm{m}$ thickness) and a concentric ring of $\mathrm{Li}$ metal $(22 \mathrm{~mm}$ inner diameter) as the reference electrode. The electrolyte was 1 $\mathrm{M} \mathrm{LiPF}_{6}$ in 1:1 EC:DEC. Electrochemical experiments were performed using a VMP2 (Bio-Logic). Cells were first cycled under galvanostatic conditions at $\mathrm{C} / 10(17 \mathrm{~mA} /$ g) between 2.5 and $4.1 \mathrm{~V}$ versus $\mathrm{Li}^{2} / \mathrm{Li}^{+}$to confirm acceptable cell performance. Impedance spectra were then collected at successive intervals of $15 \mathrm{mAh} / \mathrm{g}$ (approximately $10 \%$ SoC, given a practical capacity of about 150 $\mathrm{mAh} / \mathrm{g}$ for the electrodes). The cell was allowed to relax at OCV for $20 \mathrm{~min}$ before each impedance measurement. Impedance spectra were collected over the range of 200 $\mathrm{kHz}$ to $10 \mathrm{mHz}$ with a peak-to-peak amplitude of $15 \mathrm{mV}$.

\section{Results and discussions}

The impedance spectra were generated at time intervals corresponding to specific states of lithiation/delithiation in the electrode, which in turn influence the transport properties and hence the electrochemical response. The inset in Fig. 1 shows the pulsed constant current charging profile. The same load cycle as utilized in the experiments was adapted in the simulations, where each pulse generates a 10 $\%$ increment in the SoC from 0 to $100 \%$, and where the simulated impedance response is generated at every increment. Figure 1 shows the resulting Nyquist plot of the half-cell at various SoC levels, calculated using Eq. 14. The general features of the impedance spectra are similar, mainly displaying a large semicircle for medium frequencies and a finite diffusion branch for high frequencies. The most dominant contribution is assigned to the chargetransfer process between the LFP electrode and the electrolyte, corresponding to an increased value of $C_{\mathrm{Li}}$ in Eq. 2 for increasing $\mathrm{SoC}$ values. The strong $\mathrm{SoC}$ dependency found for the degree of lithiation in the positive electrode has also been confirmed in several experimental studies $[1,12]$. The identified charge-transfer processes there are in the same frequency range and show a similar $\mathrm{SoC}$ dependency as in the simulations, suggesting an agreement with the proposed interpretation. The impedance response can be characterized as different polarization processes in the $\mathrm{LiFePO}_{4}$ cathode: solid state diffusion or intercalation of $\mathrm{Li}$ ions (as described in Eqs. 2 and 3), charge transfer (cathode/electrolyte) described via Butler-Volmer kinetics (Eq. 6) and contact (particle) resistance described by Ohm's law (Eqs. 5-9).
Figure 2 shows the Bode modulus and phase plots, respectively, generated from the simulations and thereby the frequency dependence of the SoC. The resistance and frequency range of the different processes in the cathode can then be separated. The Bode phase plots display a suppression of the peak at $\sim 10^{2.7}-10^{3} \mathrm{~Hz}$, while the Bode modulus shows a lower resistance and a shift to higher frequencies for the diffusion onset for higher SoC values. This corresponds to higher charge-transfer and solid state diffusion resistances for higher cathode lithiation, equivalent to a higher $C_{\mathrm{Li}}$ in Eq. 3. The different time constants for both these phenomena are responsible for a constant phase element (CPE)-type behaviour (i.e. phase angles $>45^{\circ}$ ). The $\mathrm{CPE}$ behaviour indicates the exact frequency of shifting power law behaviour in the Bode magnitude plots, seen as a shift in the slope of the linear curves at ca. $10^{3.3} \mathrm{~Hz}$. The crossover frequency, where the Bode plots display at plateau at lower frequencies, is higher for higher SoC levels, indicating a smaller diffusion length for these SoC levels inside the bulk of the porous electrode. Moreover, the transition from diffusion controlled to charge-transfer-controlled responses at different frequency ranges can be observed in the simulations, in agreement with previous studies $[2,3,16,17]$ where high $\mathrm{SoC}$ is indicated through lower charge-transfer resistance.

The Warburg-like behaviour seen at higher frequencies can be attributed to several factors, but primarily to the porosity of the electrodes. For a porous insertion electrode, the arc displays a characteristic shape previously described in the literature [18-20]. This type of porous-electrode interfacial impedance response was, for example, discussed by de Levie, and results from the increasing ohmic drop experienced as the ionic current penetrates into the electrode with decreasing signal frequency. This is also known as ambipolar diffusion of ions and electrons within a

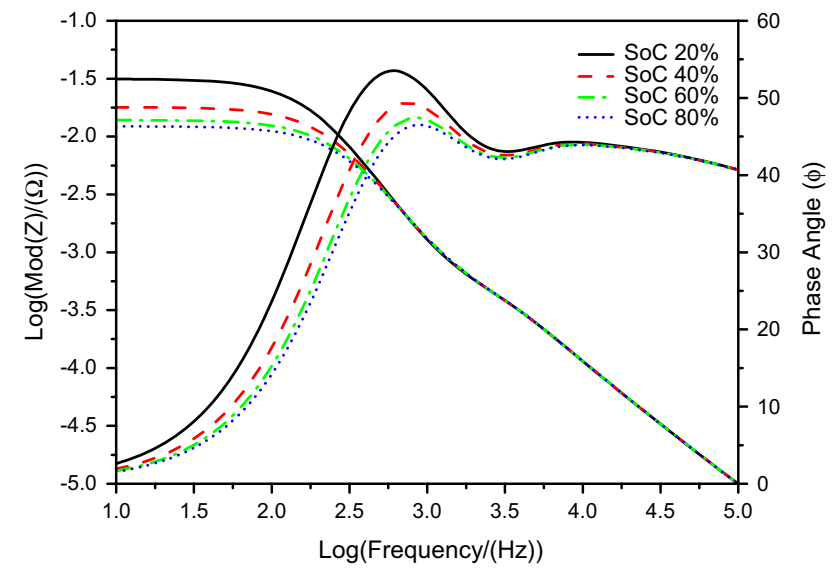

Fig. 2 Bode modulus and phase angle for various state-of-charge levels 
transmission line formed from a conductive pore of uniform diameter and a capacitive wall with a well-defined and constant area per unit length [21]. It is worth noting the similarity between this response and that of a diffusion process with a non-transmissive (blocking) interface. For a porous electrode, the current within the pore changes more sluggishly, and the penetration depth decreases with frequency. Therefore, after a very short time or at very high frequencies, primarily the capacitive effects of the more or less flat external electrode surface are measured, while the penetration distance of the sinusoidal varying current wave charging the walls of the structure is restricted at high frequencies by the resistance of the electrolyte in the pores. Figures 3 and 4 show the simulated and experimental data in Nyquist and Bode phase diagrams at various SoC levels. The Nyquist plot displays remarkably good fits between simulated and experimental EIS data, showing the validity of the model used. In the Bode plot, it can be seen that the match is striking in the frequency region characteristic of the charge-transfer regime $\left(10^{2.7}-10^{3} \mathrm{~Hz}\right)$, while the agreement is less good for higher frequencies. This is indicative of the importance of cell design parameters such as particle contacts, porosity and current collector/electrode contacts. These would appear in the high-frequency region, where the EIS response is considered to be dependent on porosity or particle-particle resistance [22-24].

To further explore this phenomenon, the effect of porosity directly correlated to the volume fraction of electrolyte in the electrode ( $\epsilon_{1}$ in Eqs. 3 and 5) is investigated. Porosity is an important parameter, as it scales the effective diffusion coefficient and conductivity as described by the porous-electrode theory [25]. Figure 5 shows Nyquist plots for 20, 40 and $60 \%$ SoC levels and different

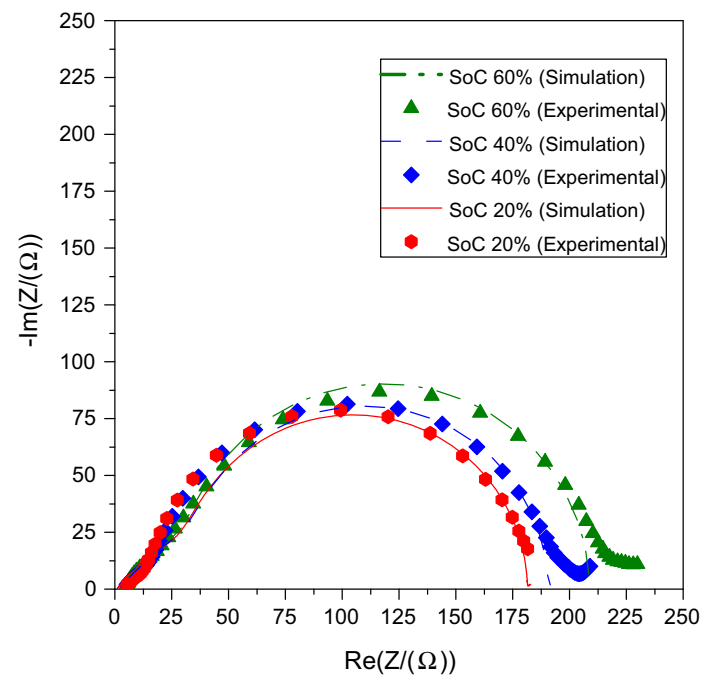

Fig. 3 Simulated and experimental Nyquist plots

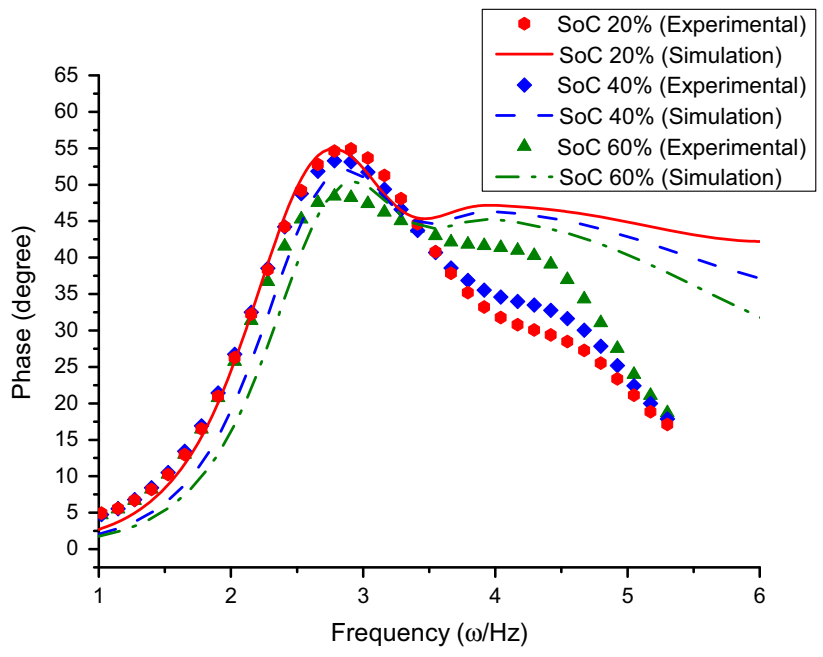

Fig. 4 Simulated and experimental Bode phase plots

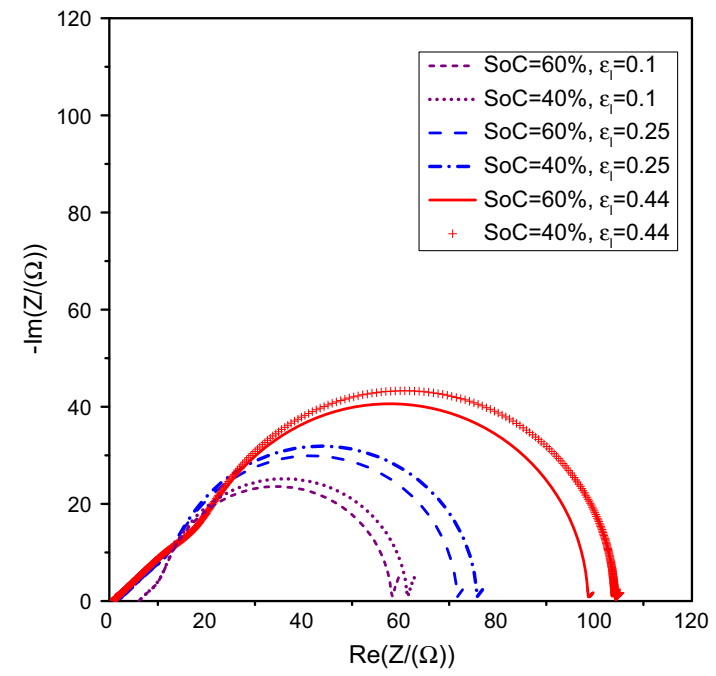

Fig. 5 Nyquist plots for various electrolyte volume fractions $\left(\epsilon_{1}\right)$ and state-of-charge levels

electrode porosities. Generally, two features can be highlighted. First, the set of plots generated for the same SoC levels yield significantly different polarization for the different electrolyte volume fractions. More explicitly, lower porosity values yields a smaller semicircle in the Nyquist plot, corresponding to a lower charge-transfer resistance. A larger volume fraction of liquid electrolyte, on the other hand, leads to higher polarization. This can be explained by that a denser packing of the particles decreases the intraparticle resistance since the overall connectivity in the electrode provides a better interface for charge transfer, lowering the polarization resistance (see Eq. 6). Secondly, the real axis impedance (high-frequency intercept), which is a signature of the ohmic resistance, shows the reverse trend, i.e. it is greater for the lower electrolyte volume 
fraction in the porous electrode and lower for the more porous electrodes. This is due to the limited ionic conduction resulting from the low electrolyte content (see Eq. 8).

Figure 6 shows the phase plot of EIS data generated for different SoC levels and different porosities $\left(\epsilon_{1}\right)$, which directly corresponds to the volume fraction of electrolyte in the electrode composite. As stated above, the porosity yields the effective transport parameters through Eqs. 3, 5, 6 and 8. High porosities will yield good electrode/electrolyte contact, which renders high "effective" diffusivity and ionic conductivity through the electrode volume, as can be seen through the transport Eqs. 5-8. It can be seen from the simulation results that the high-frequency response is more or less independent of the SoC level, i.e. one cannot sense any deviation in the high-frequency region for the $\mathrm{SoC}$ variation. However, the change in phase angle is quite dramatic when altering the electrolyte volume fraction. This shift of the phase at higher frequencies can therefore be taken as an indication of porosity changes (or porosity in general) during the course of battery cycling. This region in the Bode phase plot can hence display the effect of experimental variables such as electrode calendering, volume changes or battery packing. If comparing the simulated phase angle data with the experimental equivalents in Fig. 4, the values are most similar for electrode porosities equivalent to 0.20 in electrolyte volume fraction. This can be considered somewhat of a low porosity estimate for an uncalandered $\mathrm{LiFePO}_{4}$ electrode. We estimate the electrodes used in the experimental part of this work as being in the range of $75-80 \%$ porous, based on the ratio of the density of the coating relative to the theoretical density of a perfectly compacted non-porous electrode. This is a reasonable estimate for an uncalendered electrode with a nanosized active material. However, this estimate does not include a consideration of the electrode

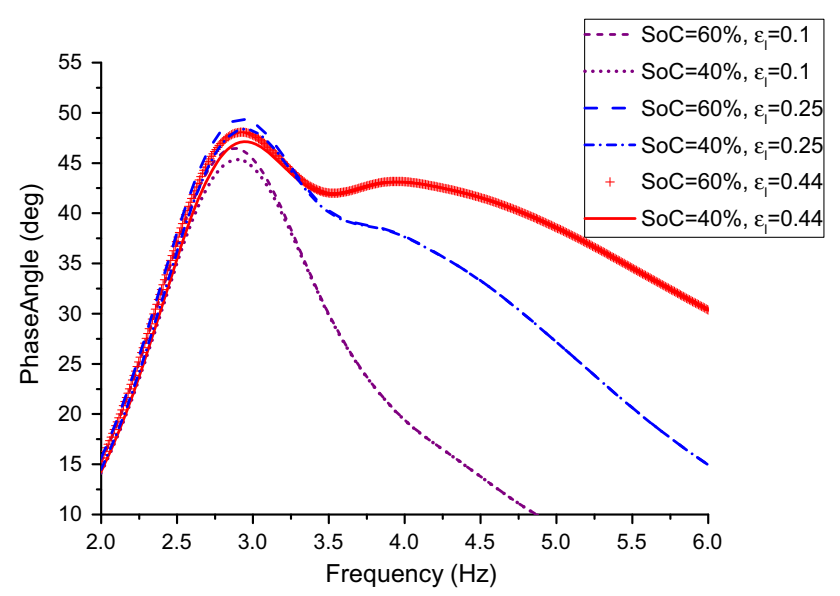

Fig. 6 Bode plots for various electrolyte volume fractions $\left(\epsilon_{1}\right)$ and state-of-charge levels tortuosity, which requires advanced techniques such as tomography to be quantified.

It is also clear from Fig. 6 that there are significant variations is this regime of the phase plots, also for constant porosities, indicating that more refined modelling is necessary to adequately capture all phenomena appearing in this frequency domain. Nevertheless, the current model clearly captures the qualitative features of the experimental curves, i.e. the plateau and the crossing of the curves when going from higher to lower SoC values (or vice versa). The porosity seems to vary substantially during cycling, in the range of $0.2-0.5$, which certainly corresponds to realistic values.

\section{Conclusions}

The relationship between SoC and EIS data has been explored for $\mathrm{LiFePO}_{4}$ half-cells by employing a multiphysics approach. It can be seen that the charge-transfer resistance is dependent of the SoC during charging, and can be modelled in good agreement with experimental results. Cell design parameters, e.g. calendering, will influence the EIS response since they control the solid state conduction path in the electrode, and the simulations thus provide information on morphological parameters. Especially at higher frequencies, the simulations can qualitatively reproduce the features of porosity changes during battery cycling. With controlled variation of electrode porosity and model refinement, this relationship can most likely be explored to a higher degree in future studies. Such an approach will be necessary for SOC diagnostics using an electrochemical modelling tool when investigating batteries with porous electrodes. The simulations here evidently show clear shifts in porosity while cycling the battery, which can be quantified by simulation data.

In summary, the FEM approach provides insight into the impedance response without the need for equivalent-circuit modelling, thereby better capturing the interplay between cell chemistry, geometry and morphology. This may ultimately yield better input parameters for adaptive BMS algorithms such as 'fuzzy logic' or Kalman filtering.

Acknowledgements The research leading to these results has received funding from the European Union's Seventh Framework Programme (FP7/2007-2013) under grant agreement $n^{\circ}$. 608575. We also acknowledge support from the Swedish Energy Agency (STEM), project P42031-1.

Open Access This article is distributed under the terms of the Creative Commons Attribution 4.0 International License (http://crea tivecommons.org/licenses/by/4.0/), which permits unrestricted use, distribution, and reproduction in any medium, provided you give appropriate credit to the original author(s) and the source, provide a link to the Creative Commons license, and indicate if changes were made. 


\section{References}

1. Blanke H, Bohlen O, Buller S, De Doncker RW, Fricke B, Hammouche A, Linzen D, Thele M, Sauer DU (2005) Impedance measurements on lead acid batteries for state-of-charge, state-ofhealth and cranking capability prognosis in electric and hybrid electric vehicles. J Power Sour 144:418-425. doi:10.1016/j. jpowsour.2004.10.028

2. Rodrigues S, Munichandraiah N, Shukla AK (2000) A review of state-of-charge indication of batteries by means of ac impedance measurements. J Power Sour 87:12-20. doi:10.1016/S03787753(99)00351-1

3. Rodrigues S, Munichandraiah N, Shukla AK (1999) AC impedance and state-of-charge analysis of a sealed lithium-ion rechargeable battery. J Solid State Electrochem 3:397-405. doi:10.1007/s100080050173

4. Fleischer C, Waag W, Heyn H-M, Sauer DU (2014) On-line adaptive battery impedance parameter and state estimation considering physical principles in reduced order equivalent circuit battery models: Part 1 Requirements, critical review of methods and modeling. J Power Sour 260:276-291. doi:10.1016/j.jpow sour.2014.01.129

5. Nakura K, Ariyoshi K, Ogaki F, Takaoka K, Ohzuku T (2014) Characterization of lithium insertion electrodes: a method to measure area-specific impedance of single electrode. J Electrochem Soc 161:A841-A846. doi:10.1149/2.090405jes

6. Ogihara N, Kawauchi S, Okuda C, Itou Y, Takeuchi Y, Ukyo Y (2012) Theoretical and experimental analysis of porous electrodes for lithium-ion batteries by electrochemical impedance spectroscopy using a symmetric cell. J Electrochem Soc 159:A1034-A1039. doi:10.1149/2.057207jes

7. Ender M, Weber A, Ellen I-T (2011) Analysis of three-electrode setups for AC-impedance measurements on lithium-ion cells by FEM simulations. J Electrochem Soc 159:A128-A136. doi:10. 1149/2.100202jes

8. Zadin V, Brandell D, Kasemägi H, Aabloo A, Thomas JO (2011) Finite element modelling of ion transport in the electrolyte of a 3D-microbattery. Solid State Ion 192:279-283. doi:10.1016/j.ssi. 2010.02.007

9. Zadin V, Kasemägi H, Aabloo A, Brandell D (2010) Modelling electrode material utilization in the trench model 3D-microbattery by finite element analysis. J Power Sour 195:6218-6224. doi:10.1016/j.jpowsour.2010.02.056

10. Dickinson EJF, Ekström H, Fontes E (2014) COMSOL multiphysics ${ }^{\circledR}$ : finite element software for electrochemical analysis A mini-review. Electrochem Commun 40:71-74. doi:10.1016/j.ele com.2013.12.020

11. Kindermann FM, Noel A, Erhard SV, Jossen A (2015) Long-term equalization effects in Li-ion batteries due to local state of charge inhomogeneities and their impact on impedance measurements. Electrochim Acta 185:107-116. doi:10.1016/j.electacta.2015.10. 108
12. Doyle M, Fuller TF, Newman J (1993) Modeling of galvanostatic charge and discharge of the lithium/polymer/insertion cell. J Electrochem Soc 140:1526-1533. doi:10.1149/1.2221597

13. Wang C, Sastry AM (2007) Mesoscale modeling of a Li-ion polymer cell. J Electrochem Soc 154:1035-1047. doi:10.1149/1. 2778285

14. Srivastav S, Tammela P, Brandell D, Sjödin M (2015) Understanding ionic transport in polypyrrole/nanocellulose composite energy storage devices. Electrochim Acta 182:1145-1152. doi:10.1016/j.electacta.2015.09.084

15. Ramadass P, Haran B, White R, Popov BN (2002) Performance study of commercial $\mathrm{LiCoO}_{2}$ and spinel-based $\mathrm{Li}$-ion cells. J Power Sour 111:210-220. doi:10.1016/S0378-7753(02)00267-7

16. Osaka T, Nara H, Mukoyama D, Yokoshima T, Momma T (2014) Keynote presentation: approaches of lithium ion batteries evaluation by means of electrochemical impedance spectroscopy. Meeting Abstracts, MA2014-03:97

17. Piller S, Perrin M, Jossen A (2001) Methods for state-of-charge determination and their applications. J Power Sour 96:113-120. doi:10.1016/S0378-7753(01)00560-2

18. de Levie R (1967) Advances in electrochemistry and electrochemical engineering. In: Delahay $\mathrm{P}$ (ed), vol 6. Interscience Publishers, New York, p 329

19. de Levie R (1963) On porous electrodes in electrolyte solutions: I. Capacitance effects. Electrochim Acta 8(10):751-780. doi:10. $1149 / 1.1393162$

20. Doyle M, Meyers JP, Newman J (2000) Computer simulations of the impedance response of lithium rechargeable batteries. J Electrochem Soc 147(1):99-110. doi:10.1149/1.1393162

21. Esterle TF, Sun D, Roberts MR, Bartlett PN, Owen JR (2012) Evidence for enhanced capacitance and restricted motion of an ionic liquid confined in $2 \mathrm{~nm}$ diameter Pt mesopores. Phys Chem Chem Phys 14:3872-3881. doi:10.1039/C2CP23687G

22. Creus J, Mazille H, Idrissi H (2000) Porosity evaluation of protective coatings onto steel, through electrochemical techniques. Surf Coat Technol 130:224-232. doi:10.1016/S02578972(99)00659-3

23. Itou Y, Ogihara N, Okuda C, Sasaki T, Nakano H, Kobayashi T, Takeuchi Y (2014) Electrochemical analysis and novel characterization of porous electrodes by using transmission line model for lithium-ion batteries. Meeting Abstracts, MA2014-04: 611. doi:10.1021/jp512564f

24. Roßberg K, Paasch G, Dunsch L, Ludwig S (1998) The influence of porosity and the nature of the charge storage capacitance on the impedance behaviour of electropolymerized polyaniline films. J Electroanal Chem 443:49-62. doi:10.1016/S0022-0728(97) 00494-4

25. Levi MD, Aurbach D (2014) Impedance of a single intercalation particle and of non-homogeneous. Multilayered porous composite electrodes for Li-ion batteries. J Phys Chem B 108:11693-11703. doi:10.1021/jp0486402 